https://doi.org/10.21670/ref.2017.37.a04

Artículos

\title{
Las fronteras, la movilidad y lo transfronterizo: Reflexiones para un debate
}

\section{Borders, mobility and the transborder space: Reflections for a discussion}

Marcela Tapia Ladino ${ }^{\text {a* }}$ (i) https://orcid.org/0000-0002-2846-710X

${ }^{a}$ Universidad Arturo Prat, Instituto de Estudios Internacionales, Chile, correo electrónico: marcelatapial@gmail.com

\section{Resumen}

El propósito de este manuscrito es hacer una aportación al debate de lo trans y lo fronterizo a partir de los estudios de las fronteras para pensar un marco explicativo que permita comprender qué convierte a una región o espacio fronterizo en transfronterizo. La metodología fue fundamentalmente revisión de la producción teórica anglo y latinoamericana al respecto. La consideración de la escala y la mirada desde el territorio en el análisis es central para comprender esta conversión. Postulamos que región o espacio fronterizo se transforma en transfronterizo fundamentalmente por la interacción que producen las movilidades de las personas en sus constantes y continuos cruces y así como por el despliegue de una serie de prácticas sociales que tienen a la frontera como referente y como recurso.

Palabras claves: frontera, movilidad, territorio, transfronterizo.

\section{Abstract}

Autor para correspondencia: Marcela Tapia Ladino, correo electrónico: marcelatapial@gmail.com

The purpose of this manuscript is to contribute to the discussion of transborder and border issues based on border studies in order to develop an explanatory framework that allows one to understand what makes a border region or space a transborder region or space. The consideration of scale and perspective from the territory under analysis is important for understanding this shift. We theorize that a region or space becomes transborder mainly through the interaction produced by the constant crossing and mobility of people as well as the deployment of a series of social practices in which the border is both a reference and a resource.

Keywords: border, mobility, territory, transborder.
Esta obra está protegida bajo una Licencia Creative Commons Atribución-NoComercial 4.0 Internacional.
CÓMO CITAR: Tapia, M. (2017). Las fronteras, la movilidad y lo transfronterizo: Reflexiones para un debate [Borders, mobility and the transborder space: reflections for a discussion]. Estudios Fronterizos, 18(37), 61-80, doi:10.21670/ref.2017.37.a04 


\section{Introducción ${ }^{1}$}

Como hemos señalado en otros trabajos (Tapia y González, 2014; Tapia y Parella, 2015) los estudios sobre fronteras han experimentado importantes cambios desde la segunda mitad del siglo $\mathrm{xx}$ a la fecha y no solo eso, sino que se han vuelto cada vez más inter y transdisciplinarios (Newman, 2015). En general los estudios de frontera han discurrido de forma paralela (Zapata-Barrero y Ferrer-Gallardo, 2012, p. 14) a los cambios históricos como la caída del muro de Berlín (1989), la consolidación y expansión de la Unión Europea, los ataques terroristas en Europa y Estados Unidos, en especial el del 9/11 (Agnew, 2008) y la reciente crisis de refugiados sirios en Europa y Medio Oriente. Todos estos hechos han llevado a debatir sobre las fronteras, sus funciones, su capacidad de cierre, apertura y de contención así como los imaginarios contenidos en la demarcación. De modo que hemos transitado de unas nociones más bien fijas y separadoras de las fronteras en clave geopolítica y decimonónica, a visiones optimistas de un mundo sin fronteras en contextos de procesos de integración (Unión Europea y Mercosur) y más recientemente al cierre de las fronteras y al surgimiento de temores en torno a quienes las cruzan y sus verdaderas intenciones. Evidentemente las contingencias históricas afectan la producción de las investigaciones sobre las fronteras, pero en ningún caso los resultados son progresivos o van en una sola dirección. A menudo persisten interpretaciones que se solapan o privilegian una visión sobre otra.

Según la bibliografía se estima que en los últimos 20 años los estudios sobre fronteras han superado los límites de disciplinas como la geografía, ciencias políticas, historia, sociología, antropología y las relaciones internacionales, entre otras (Newman, 2015). El "cruce de las fronteras" disciplinarias es una buena metáfora para remitir a la transdisciplinariedad (cross-disciplinary) de los estudios de frontera así como la profusión de publicaciones y entidades abocadas a este objeto de estudio (Newman, 2015, p. 14). ${ }^{2}$ Esta alta productividad tiene ventajas y limitaciones, las primeras se relacionan con el aumento en los estudios de casos de distintas fronteras basadas en etnografías, encuestas y explotación de datos; pero también tiene limitaciones, puesto que la naturaleza interdisciplinar del debate hace que la discusión teórica sea compleja y no se haya constituido un "subcampo científico unificado" (Brunet-Jailly, 2005, p. 634).

Como señalamos, la producción de los estudios de frontera (border studies) es amplia y considera el uso de una serie de nociones o glosario común de terminologías fronterizas, pero poco discutidas, como son las nociones: fronterizo, transfronterizo o regiones fronterizas, por mencionar solo algunos. Todos estos conceptos se utilizan a distintas escalas desde lo local a lo global hasta para aludir a categorías sociales y culturales diferentes. A partir de la experiencia investigativa que vincula frontera, migración y movilidad, el propósito de este trabajo es aportar al debate sobre lo transfronterizo o la transfrontericidad en el contexto de una región o zona fronteriza. El afán es revisar la producción sobre fronteras, tanto de la historia como los puntos de vista disciplinarios, así como aquellos que abordan lo transfronterizo desde el punto de vista de las urbes y de la movilidad humana. El objetivo es alcanzar una visión general de la discusión sobre fronteras

\footnotetext{
${ }^{1}$ Este manuscrito corresponde a los resultados de investigación del Proyecto FONDECYT Regular $\mathrm{N}^{\circ}$ 1150123 Cruzar y vivir en la frontera de Arica y Tacna. Frontera y circulación 1990-2010.

${ }^{2}$ Revisar las investigaciones y hallazgos de El Colegio de la Frontera Norte y El Colegio de la Frontera Sur de México y los trabajos publicados por la Association for Borderlands Studies (ABS) en el Journal of Borderlands Studies, por mencionar algunos.
} 
y cómo se incluye en este debate lo transfronterizo, poniendo especial interés en los movimientos de población en torno y en las fronteras. Para eso revisamos la bibliografía producida sobre fronteras y transfrontericidad, especialmente a partir de la experiencia latinoamericana, pero no exclusivamente.

Dentro del amplio debate de frontera y lo transfronterizo privilegiaremos los análisis que se ubican en los territorios y desde la perspectiva de quienes habitan la frontera, puesto que de lo contrario corremos el riesgo de confundirnos con nociones más amplias como transnacionalismo e incluso globalización. En definitiva tendremos en cuenta la noción de análisis multiescalar o multinivel como herramienta heurística para diferenciarnos de los estudios que se sitúan en la escala macro o supranacional, como los de la globalización. Esta opción no implica un descarte de lo uno por lo otro, sino por el contrario, reconocemos como señala Sassen la existencia de una jerarquía de las escalas, pero que al mismo tiempo, y como la misma autora señala, estas han sido y siguen siendo desestabilizadas. Por tanto es preciso reconocer que "lo global trasciende el marco exclusivo del Estado-nación y al mismo tiempo habita parcialmente los territorios y las instituciones nacionales" (Sassen, 2007, p. 11).

El artículo se organiza de la siguiente forma, en la primera parte revisamos el debate sobre las fronteras en los estudios de frontera (border studies) de manera sucinta con una mirada histórica, luego una revisión de los cambios de su contenido en las distintas disciplinas y las mudanzas en el debate de América Latina. La segunda parte se detiene en la producción teórica sobre lo transfronterizo para lo cual se revisan algunos trabajos que definen esta noción y la aplican a casos concretos como las eurorregiones y regiones transfronterizas latinoamericanas. En la tercera parte incluimos el debate sobre las metrópolis, complejos urbanos y conglomerados transfronterizos desarrollado a partir de casos en México-Estados Unidos, Haití-República Dominicana y Argentina-Bolivia. Para finalizar nos abocamos al vínculo entre fronteras, movilidad y prácticas sociales fronterizas como bases para comprender la transfrontericidad en las regiones fronterizas.

\section{Las fronteras en los estudios fronterizos}

\section{Las fronteras en la historia: Breve revisión}

La noción de frontera ha experimentado cambios a lo largo de la historia, asimismo posee distintos significados y diversas acepciones por disciplina. Respecto al primer punto podemos afirmar que la historia de la idea de fronteras es amplia y retrotrae a la antigüedad, pero claramente los contenidos han variado en el tiempo. Según Brunet-Jailly (2005, p. 635), durante el Imperio Romano y bajo la política de expansión, la distinción entre civilización y barbarie fue central para jerarquizar los espacios dominados. En la Edad Media el sistema feudal estuvo más preocupado por el control y defensa de los territorios y pueblos que de la delimitación de los espacios. Las fronteras eran lejanas y se desplazaron hacia las medianías del océano Atlántico. Más tarde los geógrafos, y gracias a las técnicas de mapeo, pudieron avanzar hacia una visión espacial de las posesiones y establecer los borderlands, frontier o zonas fronterizas (Brunet-Jailly, 2005).

En el siglo xvir y en un contexto de formación del orden político moderno, el reconocimiento de la soberanía de los Estados y del territorio de los Estados nación estuvo 
en el centro del Tratado de Westfalia de 1648 (Velde y Naerssen, 2011). Como señala Amilhat (2013) este tratado marcó un hito porque terminó con los afanes de dominación universal de los estados europeos y en especial porque "el orden feudal que estaba basado en la lealtad interpersonal se veía remplazado por una lógica territorial, según la cual la autoridad y la soberanía derivaban de la constitución de un Estado dotado de límites lineales" (Amilhat, 2013, p. 46). Estas ideas llegaron a su máximo desarrollo en el siglo XIX con los movimientos nacionalistas y la consolidación de la construcción de los estados modernos.

En la tradición norteamericana y bajo el influjo de Turner las fronteras fueron entendidas como espacios de movimientos con un fuerte contenido cultural y un afán civilizatorio. A partir de este influjo en la frontera norteamericana "el continuo avance de los colonos sobre tierras aparentemente baldías del oeste habría jugado un rol crucial en el desarrollo del sistema democrático norteamericano y habría sido un factor determinante en la formación del carácter nacional" (Ratto, 2001, p. 106). Así la expansión de la frontera convirtió a los inmigrantes recién llegados en norteamericanos estableciéndose como "la línea de americanización más rápida y efectiva" (Brenna, 2011, p. 27).

En América Latina los nuevos estados emancipados en las primeras décadas del siglo XIX se preocuparon menos por las demarcaciones y en general heredaron los viejos límites coloniales. En la segunda mitad del siglo xix la preocupación por las fronteras cobró fuerza en el contexto de la ampliación de los espacios vacíos internos o por el interés suscitado de las riquezas existentes en los territorios ubicados en los márgenes (González, 2007, González, 2008). En general es el siglo xix cuando se produce la mayor parte de las demarcaciones nacionales emergiendo con ello el derecho internacional en Europa y América Latina (Machado, 2005). En algunos casos esta preocupación por los espacios periféricos estuvo en la base de conflictos decimonónicos que más tarde alcanzaron su correlato en el siglo xx y xxi (Amilhat y Rouvière, 2009). En este sentido es posible afirmar que "las fronteras interestatales sudamericanas, en general, son construcciones históricas apenas centenarias" (Kralich, Benedetti y Salizzi, 2012, p. 117), ello explica los frecuentes litigios que surgen de tanto en tanto y que retrotraen a hechos ocurridos en el pasado.

\section{Las fronteras en las ciencias sociales y la geografía}

Desde el punto de vista disciplinario los estudios sobre frontera también han experimentado cambios importantes. Kolossov (2005) señala que es posible distinguir enfoques teóricos tradicionales y posmodernos. Entre los primeros se encuentra la cartografía que se detuvo en la morfología, funciones y régimen político de las fronteras concluyendo que no era posible establecer límites "naturales" que coincidieran con los físicos. Luego, a partir de la Segunda Guerra Mundial se acentuó el enfoque funcional que comprendió las fronteras como una realidad dada preocupándose por su permeabilidad. Desde el paradigma realista los principales actores de las fronteras en la escena internacional son los Estados —y desde la visión liberal estos no son los únicos ni los principales-. Dentro de este grupo la última aproximación es la que denomina "paradigma global" que presta atención a las redes internacionales que conectan a actores políticos y económicos, estatales y no estatales. Debido al desarrollo de las redes, las 
fronteras se transforman poco a poco en líneas virtuales y están siendo reemplazadas por fronteras económicas y culturales entre otras" (Kolossov, 2005, p. 612). El autor que seguimos señala que los enfoques tradicionales no pudieron encontrar respuestas a una serie de fenómenos que ocurren en las fronteras, como por ejemplo: por qué algunas zonas fronterizas, que por largo tiempo fueron pacíficas, pasaron en poco tiempo a ser focos de conflicto, o porqué los gobiernos y la opinión pública en algunos momentos fueron tan sensibles a los temas de frontera. En este contexto surgen las perspectivas posmodernas en 1980 y los aportes de la teoría del sistema mundo, el estructuralismo de Giddens y la noción de la construcción del espacio. Este conjunto de miradas ha permitido valorar el lugar de las comunidades locales territoriales más allá de la subordinación a entidades centrales, reconociendo el impacto del régimen de frontera, la formación de identidades y percepción de los vecinos (Kolossov, 2005, p. 617). Recientemente el marco de los enfoques posmodernos tenemos el de Política, Práctica y Percepción (PPP) que:

Representa un intento por sintetizar los últimos avances teóricos con los enfoques tradicionales que no ha perdido su valor práctico —en particular, el enfoque funcional. Desde esta perspectiva, el límite no es simplemente una institución jurídica diseñada para garantizar la integridad del territorio del Estado, sino un producto de la práctica social (Kolossov, 2005, p. 625).

Este enfoque converge con lo que hemos señalado en otra parte (Tapia y González, 2014), a partir de las contribuciones de la geografía y las ciencias sociales, que rescata la mirada de las fronteras desde los habitantes de las zonas fronterizas o los borderlanders. De manera poco detallada, las distintas perspectivas han pasado de la preocupación por las líneas de los mapas, su expresión en los territorios, el protagonismo de los dispositivos de control y de los Estados, a una preocupación por los habitantes fronterizos, las interacciones, los cruces y los préstamos (Zapata-Barrero y Ferrer-Gallardo, 2012). De modo que las fronteras son espacios donde se desenvuelven actores que las habitan y las cruzan con identidades distintas (tanto regional, como nacional, transfronteriza y transnacional), ubicados en posiciones diferentes, casi siempre asimétricas. Como señalan Ovando y Ramos "las áreas fronterizas son el lugar donde las identidades dominantes (El Estado y el tránsito de capitales) luchan por mantener incuestionada su hegemonía, mientras que las identidades subalternas luchan por el reconocimiento social" (Ovando y Ramos, 2016, p. 11). ${ }^{3}$

\footnotetext{
${ }^{3}$ Sin embargo somos conscientes que la relación entre fronteras y territorio hoy día es cada vez más compleja puesto que estas se pueden encontrar en sitios "territorialmente identificables" (Parker y Vaughan-Williams, 2009, p. 582) como pasos fronterizos, puertos o aeropuertos, pero no exclusivamente. En muchos casos las fronteras son menos visibles o más impalpables como los controles biométricos, controles de seguimientos de movilidad o transacciones financieras, por señalar algunas de alta sofisticación. Por no mencionar el impacto de internet y el ciberespacio que cuestionan la ubicuidad de la frontera como espacio fijo. Sin embargo, para el objetivo de este trabajo interesan especialmente los primeros.
} 


\section{El debate de las fronteras en América Latina}

Desde el punto de vista de los significados por disciplina, la categoría frontera es polisémica y en general alude a límites políticos y a discontinuidad (Bartolomé, 2008, p. 36). Así una primera distinción relevante es la de límite y frontera, la que nos remite casi automáticamente a la geografía y alude, de manera general, a una línea de separación o discontinuidad entre dos espacios que a partir del siglo xx se equiparó a frontera (Gasca, 2002). Durante el proceso de consolidación de los Estados nacionales el concepto de frontera aludió directamente a la escala nacional y al territorio como "soporte natural e inequívoco de los Estados, en tanto se entendía que el territorio (y sus fronteras) y no la cultura o las etnias era lo que definía a la nación” (Nuñez, 2014, p. 75). A partir de los contenidos de límite y frontera se construyó la idea de territorios homogéneos, cuya demarcación estaba dada por un contorno (el límite) externo y como línea de expansión interna con un carácter marcadamente geopolítico.

Estos supuestos fueron revisados en el debate latinoamericano de los años noventa, especialmente en los foros de Geocrítica, en los que se dio cuenta de los procesos de desnaturalización de las fronteras y las formas de abordar los distintos significados sociales y políticos de las mismas. Así como la consideración de los actores y las prácticas materiales y simbólicas en torno a las fronteras de acuerdo con distintas escalas (Hevilla, 1998; Hevilla y Zusman, 2008; Zusman, 1999).

Asimismo no es posible dejar fuera del análisis la frontera de México-Estados Unidos, que se ha erigido como modelo emblemático de las fronteras (Ribas, 2011, p. 17), especialmente por la desigualdad y la asimetría que contiene. A partir del estudio de dicha frontera se ha desarrollado una amplia producción sobre ese espacio y la sociedad fronteriza. Según Zúñiga (2009) la producción al respecto ha transitado de la idea de sociedad norteña, como una variante de la sociedad mexicana situada al norte del país, a frontera invadida para denunciar la presión y penetración extranjera y, más tarde, plantear la idea de defensa, de una frontera resistente, como reacción a la "invasión" del otro y a la pérdida de valores nacionales. En la década de los años ochenta y noventa la frontera fue vista como un laboratorio de la posmodernidad donde las sociedades fronterizas no se defienden o peligran, sino que se mezclan y se constituyen en fronteras híbridas (Zuñiga, 2009). Actualmente, y en consonancia con los debates de la academia anglo, existe un interés por conocer la perspectiva de los habitantes de las fronteras para captar sus propias definiciones y percepciones de habitar los espacios fronterizos; como espacios de difusión, intercambio y convivencia donde el foco está puesto en los habitantes y no en los aparatos de control estatal de los territorios.

En la actualidad la discusión latinoamericana sobre frontera tiene un desarrollo importante en la geografía, en especial en los últimos desarrollos como la geografía del poder y la geografía regional anglosajona (Kralich et al., 2012). Uno de sus principales exponentes, Alejandro Benedetti, plantea que el límite es el área de control de un territorio; "es un dispositivo lineal, producto del esfuerzo por definir con la mayor precisión posible el área que se quiere controlar para un 'nosotros', en oposición a un 'otro'" (Benedetti, 2013, p. 42). En cambio la frontera es una:

Implantación más bien puntual o areal, es construida con los dispositivos políticos, culturales, económicos y materiales, generalmente puestos sobre el 
límite, allí en el frente, para efectivizar el control ante situaciones actuales o potenciales de egreso/ingreso al territorio: es un filtro para lo que se mueve a través del límite (Benedetti, 2013, p. 42).

Bajo el influjo de la discusión más reciente en la geografía, las fronteras son entendidas como espacios en construcción, móviles y elásticos abandonando la idea de los lindes como espacios permanentes y estáticos. Asimismo han aportado al debate la inclusión de escalas intermedias recuperando la región y la regionalización para el estudio que vincula al espacio con el poder (Kralich et al., 2012). De manera similar producción brasileña sobre fronteras ha puesto de relieve la inclusión de la escala para estudiar la vasta frontera de ese país para comprender las asimetrías y complementariedades de las regiones fronterizas y ciudades gemelas (Dorfman, 2007; Marques y Machado, 2008).

La producción teórica sobre regiones transfronterizas es amplia y proviene fundamentalmente de los estudios de frontera (border studies). Las interpretaciones discurren entre aquellas que ubican lo transfronterizo en el carácter institucional de las regiones o en la institucionalización de los vínculos, hasta aquellas regiones que se vinculan o integran por contactos informales. El primer grupo de interpretaciones plantean que la emergencia de las regiones transfronterizas tiene que ver con el cambio en la noción de escala que surge en el contexto de la crisis del capitalismo y de la dimensión nacional para la resolución de dicha crisis. Por tanto:

La construcción de las regiones transfronterizas (RTF) se relaciona con los procesos generales de reescalamiento económico, político y social; ambos responden y contribuyen a la "relativización de la escala" asociada con el declive, desde inicios de los ' 80 , de la relativa coherencia estructurada entre la economía, el Estado y la sociedad nacional que había caracterizado el apogeo del boom de la postguerra (Jessop, 2004, p. 26).

Desde un punto de vista histórico las regiones o espacios transfronterizos tienen su correlato en el pasado, pero en las últimas décadas del siglo xx han proliferado gracias al declive del proteccionismo, al auge del neoliberalismo, el fin de la Guerra Fría "y los compromisos políticos hacia la integración regional" (Jessop, 2004, p. 32). Las más destacadas son aquellas que han sido objeto de políticas específicas de la cooperación y colaboración, especialmente en el marco de la consolidación de la Unión Europea (UE) y la emergencia de las Eurorregiones en la década de los noventa. En este sentido Perkmann (2003) señala que las regiones transfronterizas aluden a regiones que surgen con base en la cooperación transfronteriza, que poseen una cierta estabilización de los contactos y la creación de instituciones que organizan los vínculos a lo largo del tiempo (Perkmann, 2003). Si bien las eurorregiones son espacios institucionalizados tienen su origen en vínculos previos, en rasgos homogéneos de los territorios adyacentes a una frontera e interdependencias funcionales que motivaron la cooperación. La noción de región funcional sirve para comprender la definición de región transfronteriza porque hace referencia a "unidades territoriales caracterizadas por una alta densidad de las interacciones internas en comparación con el nivel de las interacciones fuera" (Perkmann, 2003, p. 4). Sin embargo, de acuerdo con Perkmann, la regionalidad no puede darse por sentada, sino que debe ser entendida como 
un proceso de construcción social, es decir, como un espacio socio-territorial. Lo que importa en este caso no es solo si existen similitudes étnicas, interdependencias funcionales o un pasado común, sino un proceso de construcción y en esto la cooperación transfronteriza es central para su definición.

En una línea similar Zimmerbauer (2011) señala que a partir de la experiencia europea las regiones transfronterizas son una "configuración territorial reunida y construida por diferentes estados nacionales o regiones subnacionales a partir de dos o más Estados, ya sea por sí mismos o con la ayuda de un tercero" (Zimmerbauer, 2011, p. 213). Este autor define la región transfronteriza como una unidad territorial más o menos limitada integrada por los territorios de las autoridades que participan en una iniciativa de cooperación transfronteriza, para su definición propone un modelo de análisis por capas, entre las que distingue las capas social, jurídica, económica, política y cultural.

Otro grupo de interpretación de las regiones transfronterizas proviene, fundamentalmente, de los estudios de frontera, y se ubican en aquellos que otorgan menos importancia a la institucionalidad o formalidad y atienden más a los vínculos o relaciones que se construyen en torno a las fronteras. Para Morales (2010) una región transfronteriza es un espacio de vida que traspasa las líneas de separación (límite) y origina una integración entre los territorios colindantes. Este espacio surge frente a las asimetrías generadas por el capital, el Estado y la transnacionalización que dan origen a un conjunto de prácticas sociales que tienen a la frontera como referencia (Morales, 2010, pp. 189-190). En el centro de la definición se ubica la vecindad como elemento central y un conjunto de prácticas sociales que, a diferencia de otras localidades del país, tienen a la frontera en el centro. Estas son las que dan sentido a este espacio y contribuyen a su transformación, por tanto, para el autor son las "prácticas que despliegan las personas respecto de la frontera lo que convierte a una región (natural e histórica) en una región transfronteriza" (Morales, 2010, p. 191).

En un sentido similar Newby (2006), de acuerdo con una investigación hecha en la frontera de Estados Unidos y México, define la región transfronteriza como un modo de vida caracterizado por la continua interacción entre individuos que pertenecen a dos estructuras socioeconómicas y que comparten una frontera en común. De modo que lo transfronterizo se refleja en las transferencias de movimiento y el uso del espacio fronterizo, como viajes y compras. Los movimientos internacionales están inmersos en estructuras locales de los países que comparten frontera así como la estructura de la región de frontera. Por tanto sus habitantes deben aprender a negociar las distintas oportunidades y limitaciones específicas de la vida fronteriza y las diferencias de aquellas en el interior de cada país, esto puede dar como resultado una forma de pensar o una "sociedad fronteriza" (Newby, 2006). Este autor señala que "la región transfronteriza es tanto una línea divisoria entre los estados-nación, y un recordatorio de las consecuencias sociales del proceso de frontera definición y redefinición" (Newby, 2006, p. 3).

En un sentido similar Durand (2015) señala que la integración transfronteriza viene a ser el motor de la producción del espacio transfronterizo en un contexto en que los límites ponen de manifiesto la relación paradójica entre enfoques geopolíticos y sociales en las fronteras. El autor señala que la noción de espacio transfronterizo adquiere sentido a partir de los aportes de Lefevre quien define el espacio como construcción social. Por tanto, el espacio transfronterizo es "el resultado de interacciones y retroacciones, pasadas y presentes, el producto de todo tipo de encuentros: desde la 
planificación de material y financiera a la puesta en red de los actores, de las relaciones sociales en la definición de las estrategias políticas" (Durand, 2015, p. 310). En este sentido Durand sostiene que el espacio transfronterizo es producto de factores contextuales que favorecen la interacción por un lado, así como los procesos de refronterización (rebordering) y des-fronterización (de-bordering) del límite, por otro. Por tanto el contexto funciona como un espacio estructurador de la producción del espacio transfronterizo donde juegan un rol importante las transformaciones económicas y sociales, las reconfiguraciones territoriales y las percepciones y las prácticas de los individuos. De modo que un ambiente desfavorable para la cooperación y la interacción limitará la integración fronteriza y por tanto la producción de espacio transfronterizo. Mientras que lo contrario, es decir, factores contextuales favorecedores como una situación geopolítica pacífica, disposición de los actores a cooperar y marcos jurídicos que permitan una coordinación espacial e institucional para aumentar los intercambios entre otros, facilitarán la producción de un espacio transfronterizo.

\section{Ciudades, metrópolis y conglomerados transfronterizos}

Otro ámbito de la discusión se refiere a la expresión de lo transfronterizo en las ciudades, metrópolis o conglomerados urbanos situados en las fronteras. Se trata de estudios empíricos a partir de casos de ciudades gemelas que discuten la naturaleza transfronteriza o no de ciudades próximas. Los casos más notables son los de las ciudades de México y Estados Unidos y uno de los autores más prolíficos en esta producción ha sido Tito Alegría (2008). A partir de los estudios sobre Tijuana y San Diego iniciada en los años ochenta del siglo pasado, y de los estudios de Herzog (Hergoz como se citó en Alegría, 2008), Alegría debate la existencia de una metrópolis transfronteriza para este caso. Para eso cuestiona a autores como Bustamante (citado en Alegría, 2008) y debate los supuestos de la naturaleza binacional de las ciudades, la existencia de la misma estructura social a través de las fronteras y las similitudes en los procesos económicos y sociales a uno y otro lado del linde (Alegría, 2008, p. 131). De manera similar critica la noción de metrópoli transfronteriza de Herzog quien postula que para entender las ciudad de la frontera hay que tener en cuenta dos aspectos "el primero es el proceso de convergencia/integración o divergencia/independencia de las dos sociedades que se juntan en la frontera: el segundo es la posibilidad y necesidad de políticas elaboradas de manera binacional para las ciudades fronterizas" (Alegría, 2008, p. 133). Para este autor la frontera tiene dos tipos de funciones antagónicas y unificadoras, las primeras separan y las segundas unen a las regiones de la frontera, estos últimos son los que subyacen en la noción de metrópoli transfronteriza. En esta propuesta Herzog concluye que hay un sistema social transnacional que crea una comunidad de intereses en torno a la frontera (Alegría, 2007). Por tanto, la interacción promovida por la globalización, la sociedad red, la hibridación es la que genera integración y la emergencia de una metrópolis transfronteriza, como es el caso de Tijuana-San Diego. Así la noción de metrópolis transfronteriza es útil para definir a otros pares binacionales de ciudades fronterizas vecinas (Alegría, 2008).

Tito Alegría discute la noción de región binacional y la tesis de Herzog porque confunde espacio económico con región y postula que el intercambio transfronterizo de bienes y servicios, que da sentido a esta región, no es suficiente para definir a una 
región transfronteriza. Para el autor una región es una construcción social objetiva y su contenido:

Está determinado por un conjunto de relaciones sociales estructurales y territorializadas. Cada relación tiene un ámbito territorial que incluye la localización de los agentes y medios directamente vinculados por la relación, así como los senderos de los flujos materiales que la realizan (Alegría, 2000, p. 95).

Por tanto para identificar una región objetiva el fenómeno debe estar "objetivamente regionalizado, es decir, acotado en el tiempo y en el espacio" (Alegría, 2000, p. 95). De modo que para que la noción "continuidad transfronteriza" tenga asidero teórico son necesarios tres supuestos, según Alegría:

i) Los actores sociales de las colectividades en ambos lados de la frontera conocen procedimientos de acción similares. ii) Los actores sociales de ambos lados de la frontera interpretan y aplican del mismo modo los aspectos semánticos y normativos de esos procedimientos de acción. iii) Los actores sociales de ambos lados de la frontera tienen acceso al mismo tipo de recursos. Pero, como lo indica la evidencia, estas condiciones de estructuración social no se reúnen en la frontera (Alegría, 2008, p. 137).

Para el caso de Tijuana-San Diego, Alegría concluye que los procedimientos de acción no son similares a ambos lados de la frontera, que no hay conocimientos compartidos y en ese sentido el idioma es un obstáculo para la multiplicación de las prácticas sociales. No se trata de compartir prácticas acotadas en el tiempo y el espacio sino también de una interpretación compartida de las prácticas sociales que "producen un sentido del orden social que se materializa en significados. De esta manera, la construcción del sentido es intrínseca a las interpretaciones compartidas, sin depender de un ente externo" (Alegría, 2008, p. 139). Para el caso que el autor estudia la negociación de significados a ambos lados de la frontera no es frecuente sino esporádica y se centra en aspectos particulares del intercambio. Respecto de la continuidad transfronteriza, Alegría sostiene que esta implica que debe existir a ambos lados de la frontera, es decir, que las condiciones que permiten la reproducción del sistema operan indistintamente a ambos lados de la frontera. Por tanto las ciudades estudiadas están "juntas, pero no revueltas" porque no están integradas de manera sistémica por lo que:

La hipótesis de la metrópolis transfronteriza tiene el supuesto implícito de que Tijuana y San Diego conforman una unidad sistémica, es decir, que los cambios que ocurren en las actividades principales de una ciudad afectan a la otra. Puesto en términos del crecimiento, ello significa que los impulsos que hacen crecer a la otra y que los ritmos de crecimiento de ambas ciudades son similares. Sin embargo, las evidencias contradicen ese supuesto (Alegría, 2008, p. 143).

La tesis de Alegría es discutida por Dilla (2015) para quién la reticencia del autor para aceptar la existencia de sistemas urbanos transfronterizos se sustenta en tres supuestos. La noción de sistema que Alegría propone está basada en un cuerpo teórico que él critica, es decir, que "la formulación biológica y armónica que subyace en la noción funcionalista de sistema" (Dilla, 2008a, p. 21). Desde ese punto de vista 
ninguna aglomeración urbana al margen de su interdependencia podría constituirse en un sistema. También critica la visión de la realidad descrita por Alegría como un escenario imaginado donde prima "una fusión equitativa" (Dilla, 2008a, p. 21) y según la cual las interdependencias entre ciudades basadas en asimetrías son leídas como una desviación. Por último objeta los principales requisitos que Alegría identifica como centrales para definir la transfrontericidad como la interpretación de prácticas sociales compartidas, la existencia de códigos comunes, incluso el idioma. Dilla sostiene que esos requisitos son válidos para cualquier ciudad dentro de un mismo espacio nacional. En un sentido similar los estudios brasileños sobre ciudades gemelas postulan que las asimetrías y las diferencias en los espacios fronterizos son los que promueven la interacción laboral, los flujos de capital, el acceso a recursos y servicios de consumo colectivo (Machado, 2005; Marques y Machado, 2008), entre otros.

A partir de estas críticas Dilla propone la noción de Conglomerados Urbanos Transfronterizos (CUT) para aludir a aglomeraciones que constituyen sistemas "pero que a diferencia de otras elaboraciones, los entiende como construcciones sociales basadas en escenarios muy contradictorios e incluso conflictivos" (Dilla, 2008b, p. 169). Estos complejos funcionan en contextos de contradicción debido a las asimetrías y diferentes modos de intercambio desigual, lo que puede generar "fuertes tensiones políticas y culturales" (Dilla, 2008a, p. 22). En este sentido la propuesta de Dilla aporta al debate la inclusión de las ganancias diferenciales en los intercambios binacionales y el surgimiento de subsistemas diferenciados, lo que no es contradictorio con la idea de la formación de un sistema. Sin embargo, advierte que para que exista un cuT debe haber a lo menos seis condiciones: cercanía geográfica entre centros urbanos, reproducción económica interdependiente, existencia de relaciones primarias (de amistad, vecindad o parentesco), servicios compartidos formales e informales, relacionamientos institucionales estatales y sociales y una "percepción compartida de necesidad mutua" (Dilla, 2008b, p. 170). Dilla elabora esta propuesta a partir del estudio de ciudades fronterizas de República Dominica y Haití donde la frontera separa y pone en contacto a dos partes asimétricas y desiguales en términos económicos, institucionales y culturales. En su estudio concluye que la noción de cuT es una nueva manera de mirar el relacionamiento entre ciudades, la presión sobre los recursos, la migración y el comercio distinguiendo en cada caso si las ciudades analizadas constituyen o no un CUT.

El aporte al debate de los estudios de ciudades fronterizas enriquece la discusión de lo transfronterizo y al mismo tiempo lo objetiviza. Por una parte los parámetros de similitud, afectación mutua y códigos compartidos puede ser parte de la constitución de ciudades o metrópolis transfronterizas como señala Alegría. Sin embargo los ejemplos más frecuentes que encontramos en la realidad es que son las desigualdades y las asimetrías entre urbes fronterizas próximas las que favorecen el intercambio, la interacción y la producción de dependencia mutua, como los CUT planteados por Dilla. En ambos casos encontramos parámetros y dimensiones de nivel meso (relaciones económicas, convenios, flujos de población, etc.) para comparar o a tener en cuenta, a la hora de definir o no del carácter transfronterizo de las ciudades fronterizas. 


\section{Frontera, movilidad y territorio: La transfrontericidad y las prácticas fronterizas}

Como hemos señalado la noción de frontera ha experimentado cambios en el marco de los estudios fronterizos, sin embargo para nuestros propósitos, nos focalizamos en aquellos estudios que se ubican en la intersección entre frontera y movilidad humana. Como señalamos antes, los estudios sobre frontera han dejado de situarse en la preocupación "por el trazado, la demarcación y la localización, para sin renunciar a lo precedente, pasar a colocarse en las prácticas de (re)producción social de la frontera" (Zapata-Barrero y Ferrer-Gallardo, 2012, p. 13). La tensión que produce la movilidad humana en el cruce de la frontera es el aspecto más notorio de esta relación, procesos de cierre o endurecimiento -re-fronterización (re-bordering) — o apertura —des-fronterización (des-bordering) (Durand, 2015) son las principales preocupaciones y tensiones que definen las políticas de migración y circulación así como la percepción sobre el rol de las fronteras.

Para nuestro trabajo la opción de vincular frontera y movilidad, desde la mirada de los territorios, se relaciona con la búsqueda de la transfrontericidad en las zonas o regiones fronterizas a nivel local o micro. Sabemos que desde un punto de vista macro y en el contexto de la globalización, la mayoría de los países poseen vínculos con otros, los más evidentes son las relaciones diplomáticas y las relaciones económicas. A nivel global, la mayor expresión de estos vínculos está constituida por la acción de "organizaciones manifiestamente globales como la OIT (Organización internacional del Trabajo), los mercados financieros globales, el nuevo cosmopolitismo y los tribunales penales internacionales" (Sassen, 2010, p. 22). Pero como señala Sassen existen otros procesos que no corresponden a la escala global como tal, pero que forman parte de ella. Se trata de procesos que ocurren en los territorios, en el dominio de lo que denominamos nacional, que si bien se encuentran localizados allí, forman parte de la globalización. "Se trata de redes y configuraciones multifacéticas y transfronterizas que en algunos casos incluyen órdenes normativos y que conectan procesos, instituciones y actores subnacionales o 'nacionales', pero no necesariamente mediante el sistema formal interestatal" (Sassen, 2010, p. 22). De alguna forma postulamos que lo transfronterizo viene a ser una escala de menor rango que se vincula con lo nacional y lo global, pero que se ancla en un territorio. Esta escala es afectada por las interacciones que ocurren en torno a las fronteras, que en ocasiones son expresiones de la globalización y en no pocas ocasiones, manifestaciones de lo nacional. En esa escala territorial las personas son centrales para explicar la interacción y los vínculos que se producen al cruzar la frontera, hacer de ella un recurso y, en muchos, casos una oportunidad. Estos intercambios se dinamizan por la existencia de la frontera, gracias a ella y en ocasiones a pesar de ella, de modo que es la frontera, en su expresión territorial, la que actúa como referencia para dichos vínculos.

La categoría territorio ha sido ampliamente discutida especialmente en las últimas décadas y en el marco de la globalización, sin embargo en esta parte nos remitiremos al debate que lo relaciona con las fronteras y la movilidad. De manera sintética, nos enfocamos en la noción de territorio puesto que la frontera, lo fronterizo y lo transfronterizo se relacionan con un espacio material o espacio físico que tiene acceso controlado de personas, mercancía o bienes y servicios (Haesbaert, 2013, p. 18); por tanto, lo que hace diferente a un territorio de un área, espacio o lugar es su asociación directa con el poder y el control, tanto de los recursos o como de las personas. 
Como señala Haesbaert "de manera más simple, el territorio sería una dimensión del espacio cuando el enfoque se concentra en las relaciones de poder" (Haesbaert, 2013, p. 20); de modo que "el territorio no lo identifica y delimita el observador externo que se dispone a estudiarlo, sino los grupos sociales que mantienen relaciones de producción o reproducción, de vecindad o parentesco, etc." (Kralich et al., 2012, p. 114). Evidentemente las fronteras, desde su configuración por los Estados nacionales, poseen una función territorializadora en términos de defensa de un espacio soberano, marcador de identidad y la memoria histórica, símbolo de autoridad y control de la sociedad al actuar como filtro de las personas que entran y salen (Durand, 2015, p. 312); sin embargo, el afán de atraer capitales y bienes y participar del mercado internacional exige una redefinición del carácter regulador de las fronteras que no siempre va aparejado al libre tránsito de personas.

Para la compresión del territorio y en especial, del espacio fronterizo, la inclusión de la escala es fundamental porque el poder está desigualmente repartido en el territorio y es diferente si quienes lo ejercen son los habitantes de las fronteras, las instituciones locales o dispositivos estatales, esto es especialmente evidente en estos espacios puesto que las fronteras concretizan la territorialidad (Kralich et al., 2012, p. 115). Por esta razón la consideración de la escala también se relaciona con el tipo de poder, si lo miramos desde el Estado y en un sentido tradicional "el territorio es un macroterritorio básicamente vinculado a las grandes estructuras político-económicas dominantes" (Haesbaert, 2013, p. 26); pero si lo pensamos como resistencia, siempre presente en las relaciones sociales y que nos advierte de la capacidad de agencia, entonces tendremos "microterritorios y habrá muchas otras formas de reconstruir el poder y el territorio a partir de esta concepción" (Haesbaert, 2013, p. 26). A partir de esta última mirada estudios más recientes en el ámbito de la geografía prestan especial atención "a las formas en que los grupos sociales se identifican y manifiestan sus solidaridades a través del espacio" (Kralich et al., 2012, p. 114), de modo que el territorio es una categoría heurística definida a partir de prácticas culturales y materiales de la sociedad.

El segundo elemento a incorporar en nuestra discusión señala que en la actualidad existe bastante acuerdo en que la categoría frontera "está más vinculada a la movilidad y no tanto a una realidad estática, inamovible e innegociable, basada en una línea física territorial" (Zapata-Barrero y Ferrer-Gallardo, 2012). Como señala Benedetti la frontera alberga la hipótesis del cruce (Benedetti y Salizzi, 2011, p. 151), es decir cobra sentido en la medida que hay intención de cruzarla o de moverse a través del límite. En otros trabajos (Tapia, 2014; Tapia y Parella, 2015) señalamos que la noción de migración no es suficiente para comprender los movimientos de población que ocurren en las fronteras. El carácter bidireccional contenido en el concepto (origen-destino), el establecimiento en el lugar de destino y el tiempo de estancia, casi siempre prolongado, dejan fuera de esta noción una serie de movimientos que hasta hace poco eran tipificadas como "ambigüedad" migratoria (Cortés, 2009, p. 38) o residuales de la migración (Mallimaci, 2012).

Las fronteras son escenarios de una serie de movimientos expresados en cruces diarios, semanales o por días que no tienen por objetivo el establecimiento en el "otro lado”. Por lo tanto, la noción de movilidad es más apropiada para comprender los movimientos en torno o través de las fronteras porque "es más amplia y comprende más aspectos que la migración” (Heyman, 2012, p. 427). Las disparidades económicas y las asimetrías entre países convierten a las fronteras en un recurso, en la medida que el cruce proporciona ganancias que no se obtienen en el territorio nacional, o por lo 
menos no en la misma proporción. Así "el límite, la frontera y la movilidad devienen recursos, para definir estrategias de reproducción a partir del aprovechamiento de las disparidades entre territorios" (Benedetti y Salizzi, 2011, p. 151).

Abelardo Morales acuñó la noción prácticas sociales transfronterizas para aludir a las actividades que tienen el cruce de la frontera como elemento central. Estas prácticas pueden ser adaptativas, transformativas, productivas o reproductivas y en general hacen referencia a las ganancias, ahorro o disminución de los costos de la vida para los habitantes fronterizos que se adaptan y transforman la vida en la frontera (Morales, 2010). En un sentido similar Parella (Parella, 2014) identifica una serie de prácticas en la frontera de Mexicali-Caléxico que tienen por objeto sacar rendimiento de las ventajas de vivir de un lado y trabajar del otro, cruzar para comprar, cuidar, comer, celebrar o estudiar (Tapia y Parella, 2015). La bibliografía está llena de ejemplos de cruces con fines de consumo, tráfico, transhumancia, ocio, salud, comercio (legal-ilegal, formal-informal) en América Latina (Aponte, 2012; Hevilla, 2014; Jaquet, 2008; Mallimaci, 2012; Steiman, 2012) y en distintas latitudes (Banerjee y Chen, 2013; Shen, 2014; Soriano y Fuentes, 2015; Wang, 2004) que remiten a desplazamientos cortos cuyo objetivo no tiene que ver con quedarse del todo, sino por periodos cortos y cuyos propósitos se definen con referencia al lugar de origen.

Como señala Konrad (2015) las fronteras están en movimiento y las teorías tienen que alinearse a este "giro" en las ciencias sociales. El movimiento está fuertemente relacionado con la interacción, flujos, reubicaciones y prácticas de cruce incluso en contextos de serios conflictos o controversias. Es preciso cambiar la mirada, dejar de ver la línea para centrarnos en la interacción dinámica que se produce en la frontera, en especial a partir de la movilidad. Como advierte el autor, la reciente producción sobre fronteras muestra que "el movimiento se está convirtiendo en un amplio campo de investigación intelectual en las ciencias sociales” (Konrad, 2015, p. 4).

\section{Un aporte al debate de lo transfronterizo a modo de conclusión}

A partir de la revisión hecha y de la experiencia investigativa acumulada postulamos que la continuidad y la estabilidad de los cruces fronterizos de personas a través del tiempo, en un espacio acotado y basado en estrategias de reproducción de la vida o en actividades productivas, están en la base de la constitución de un espacio o región transfronteriza. Desde su concepción moderna, el diseño de las fronteras fue pensado en clave de cierre y clausura, tanto para proteger y contener, como para evitar dejar pasar a personas, bienes o mercancías no deseables. En torno a ellas se enfatizó el carácter nacional y el diseño de políticas de endurecimiento o apertura de las fronteras a distintas escalas, donde el control ha jugado un rol central en el ingreso, tránsito y salida.

La producción científica sobre fronteras, regiones fronterizas y espacios o regiones transfronterizas es creciente. En este aumento la preocupación de distintas disciplinas enriquece el análisis y al mismo tiempo abre nuevos desafíos teóricos y metodológicos. Por una parte, cualquier estudio sobre las fronteras debe incorporar varias miradas y por tanto diversas disciplinas, así que la transdisciplinariedad es fundamental para comprender lo que allí ocurre. Sin embargo, postulamos que la inclusión de la historia nacional y regional son claves para comprender el contexto y los cambios de idea 
de frontera en su devenir. La incorporación de la larga duración en los análisis permite comprender el resurgimiento o reavivamiento del nacionalismo y los procesos de endurecimiento y cierre de las fronteras.

A partir de la revisión de la producción sobre fronteras podemos encontrar elementos útiles para comprender qué convierte a una región fronteriza en transfronteriza. Por una parte la proximidad de poblaciones o ciudades es primordial, pero no condición suficiente. Son los intercambios, los cruces frecuentes de personas en ambos sentidos, los factores explicativos de esta configuración. La continuidad transfronteriza o las similitudes pueden ser un factor por considerar, como es el caso de las eurorregiones, pero por el contrario, las diferencias y por sobre todo las asimetrías, son los factores explicativos más relevantes para explicar el aumento de los cruces, o los intentos por cruzarla. Los casos más emblemáticos son el caso de la frontera mexicano estadounidense y, de un tiempo a la fecha, el borde externo de la Unión Europea. Sin embargo, no son casos exclusivos, a distinta escala y en distintos rincones de las fronteras de América Latina, en Asia y Europa se registran fenómenos locales de circulación y movilidad fronteriza mediante el despliegue de prácticas fronterizas productivas y reproductivas que buscan aprovechar las ventajas del cruce. Las diferencias de desarrollo a menudo se traducen en diferencias cambiarias que propician el paso al otro lado para trabajar, comprar o acceder a recursos que no se obtienen en el origen; en este sentido postulamos que lo transfronterizo se constituye desde abajo, desde el territorio, por las personas que habitan el territorio y que a menudo se mueven a través de la frontera con frecuencia independientemente de los litigios bilaterales. El despliegue de distintas prácticas sociales fronterizas convierte a la frontera en un recurso más allá de su configuración como contención o resguardo. Por tanto, más allá de las restricciones en el ingreso, la permanencia o la salida de un territorio nacional a otro, las personas buscarán resolver problemas o ganar recursos cruzando el límite siempre que sea posible; mientras en esa búsqueda van configurando territorialidades particulares y características de las zonas, comunidades y ciudades ubicadas en la frontera o en torno a ellas.

Las explicaciones que dan vida a estos espacios son variadas y así da cuenta la bibliografía, desde el consumo fronterizo, la existencia de mercados laborales fronterizos, las brechas de desarrollo que se expresan en los tipos de cambios, los servicios de todo tipo (salud, ocio y educación) y las actividades lícitas o ilícitas de tipo formal e informal, son algunos de los factores explicativos. Si bien en el caso Europeo el surgimiento de las regiones transfronterizas tiene sus antecedentes a mediados del siglo xx su expansión se verifica a fines del siglo, pero su continuidad se relaciona más con la dimensión institucionalizada de ese vínculo que formaliza vínculos territoriales preexistentes y con la intensidad de la cooperación transfronteriza. Sin embargo, sin interacciones previas, intereses compartidos o reconocimiento de dependencia mutua, difícilmente se habrían podido configurar las eurorregiones más allá de la voluntad política.

Así postulamos que son las movilidades las que construyen el territorio, que unen espacios y ciudades fronterizas que crean la transfrontericidad o lo transfronterizo, a nivel territorial. Incluso a pesar de la existencia de límites y las dificultades que en ocasiones representa el cruce. Sin embargo, se abre un desafío metodológico que consiste en verificar o validar lo transfronterizo en las regiones o espacios fronterizos. Es decir, es preciso mensurar de alguna forma cuál es la magnitud de los intercambios y cómo se constituye la interacción. Más allá de verificar las prácticas transfronterizas que hemos 
identificado en otros trabajos (Tapia, 2014; Tapia y Parella, 2015) y en la revisión hecha más arriba, es preciso no dar por sentada la transfrontericidad en una región fronteriza. Haciendo un parangón con lo que advirtieron Portes, Guarnizo y Landolt (2003) con el transnacionalismo migrante, no es suficiente "invocar algunas anécdotas" de los migrantes para hablar de transnacionalismo, sino establecer algunos mínimos. Una vez revisada la producción sobre el tema y con base en la experiencia investigativa podemos señalar que la continuidad de las interacciones en el tiempo; la magnitud de los intercambios, donde se involucre a una cantidad de población relevante en términos estadísticos y la proximidad geográfica, cuya expresión se manifiesta en movilidad en un territorio, son tres elementos centrales a considerar. Los factores explicativos pueden variar como la continuidad transfronteriza expresadas en similitudes sistémicas como discutió Alegría (2000), así como las asimetrías o desigualdades de uno y otro lado de la frontera, como plantea Dilla (2008a). Los vínculos entre gobiernos subnacionales o la cooperación transfronteriza claramente favorecen la interacción, pero planteamos que no necesariamente son condición para la interacción.

\section{Referencias}

Agnew, J. (2008). Borders on the Mind: Re-framing Border Thinking. Ethics $\mathcal{E}$ Global Politics, 1(4), 175-191.

Alegría, T. (2000). Juntos pero no revueltos: ciudades en la frontera México-Estados Unidos. Revista Mexicana de Sociología, 62(2), 89-107.

Alegría, T. (2007). Débat sur la métropole transfrontalière: une remise en cause à partir du cas Tijuana/San Diego. Cahiers des Amériques Latines, (56), 63-82.

Alegría, T. (2008). ¿Existen las metrópolis transfronterizas?: El caso de Tijuana/San Diego. En H. C. Dilla (Coord.), Ciudades en la frontera. Aproximaciones críticas a los complejos urbanos transfronterizos (pp. 129-165). Santo Domingo, República Dominicana: Editora Manatí.

Amilhat, A. L. (2013). Cultura de fronteras. En B. E. Nates (Ed.), Frontera, fronteras (pp. 43-60). Manizales, Colombia: Universidad de Caldas.

Amilhat, A. L. y Rouvière, L. (2009). Des dynamiques transfrontalières au bilan d'aménagement du territoire: Innovations et blocages dans les Andes centrales (Chili-Pérou-Bolivie). Reveu Mosella, (32), Especial Fronteras y Desarrollo, 181-196.

Aponte, J. (2012). Comercio y ocio en la transformación del espacio urbano fronterizo de Leticia y Tabatinga. En C. Zárate (Ed.), Espacios urbanos y sociedades transfronterizas en la Amazonía (pp. 205-235). Leticia, Colombia: Universidad Nacional de Colombia.

Banerjee, P. y Chen, X. (2013) . Living in In-Between Spaces: A Structure-Agency Analysis of the India-China and India-Bangladesh Borderlands. Cities, 34, 18-29.

Bartolomé, A. (2008). Fronteras estatales y fronteras étnicas en América Latina. Notas sobre el espacio, la temporalidad y el pensamiento de la diferencia. En L. Velasco (Ed.), Migración, fronteras e identidades étnicas transnacionales (pp. 35-77). México: Miguel Ángel Porrúa, Colegio de la Frontera Norte.

Benedetti, A. (2013). Los espacios fronterizos binacionales del sur sudamericano en perspectiva comparada. Revista GeoPantanal, 8(15), 37-62. 
Benedetti, A. y Salizzi, E. (2011). Llegar, pasar, regresar a la frontera. Aproximación al sistema de movilidad argentino-boliviano. Transporte y Territorio, (4), 148-179.

Brenna, J. E. (2011). La mitología fronteriza: Turner y la modernidad. Estudios Fronterizos, 12(24), 9-34.

Brunet-Jailly, E. (2005). Theorizing Borders: An Interdisciplinary Perspective. Geopolitics, 10(4), 633-649.

Cortés, G. (2009). Migraciones, construcciones transnacionales y prácticas de circulación. Un enfoque desde el territorio. Párrafos Geográficos, 8(1), 35-53.

Dilla, H. (2008a). Las ciudades en las fronteras: introducción a un debate. En H. Dilla (Coord.), Ciudades en las fronteras. Aproximaciones críticas a los complejos urbanos transfronterizos (pp. 17-29). Santo Domingo, República Dominicana: Editora Manatí.

Dilla, H. (2008b). Los complejos urbanos transfronterizos en la frontera. En H. Dilla (Coord.), Ciudades en la frontera. Aproximaciones críticas a los complejos urbanos transfronterizos (pp. 167-204). Santo Domingo, República Dominicana: Editora Manatí.

Dilla, H. (2015). Los complejos urbanos transfronterizos en América Latina. Estudios Fronterizos, 16(31), 15-38.

Dorfman, A. (2007). Fronteira e contrabando em Santana do Livramento (BR)-Rivera (Uy). Boletim Gaúcho de Geografia, (32), 75-92.

Durand, F. (2015). Theoretical Framework of the Cross-border Space Production-The Case of the Eurometropolis Lille-Kortrijk-Tournai. Journal of Borderlands Studies, 30(3), 309-328.

Gasca, J. (2002). Espacios transnacionales. Interacción, integración y fragmentación en la frontera México-Estados Unidos. México Distrito Federal: Universidad Nacional Autónoma de México, Miguel Ángel Porrúa Librero-Editor.

González, S. (2007). La emergencia de la triple-frontera andina: Perú, Bolivia y Chile. En S. González, G. Aranda, J. A. Morandé, y O. Stahringer (Eds.), Cátedras de Integración Andrés Bello. Serie Integración social y fronteras (pp. 15-54). Bogotá, Colombia: Convenio Andrés Bello.

González, S. (2008). La llave y el candado: el conflicto entre Perú y Chile por Tacna y Arica (1883-1929). Santiago de Chile: Lom.

Haesbaert, R. (2013). Del mito de la desterritorialización a la multiterritorialidad. Recuperado de http://www.culturayrs.org.mx/revista/num15/Haesbaert.pdf

Hevilla, M. C. (1998). El estudio de la frontera en América. Una aproximación bibliográfica. Revista Bibliográfica de Geografía y Ciencias Sociales, (125), 1-12.

Hevilla, M. C. (2014). Instituciones de control, familias y transhumancia en las fronteras andinas argentino-chilenas (1996-2013). Scripta Nova. Revista Electrónica de Geografia y Ciencias Sociales, XVIII(493), 1-19.

Hevilla, M. C. y Zusman, P. (2008). Diez años de estudios de fronteras en los coloquios internacionales de Geocrítica. Scripta Nova. Revista electrónica de Geografía y Ciencias Sociales, XII(270). Recuperado de http://www.ub.edu/geocrit/sn/sn-270/ sn-270-150.htm

Heyman, J. (2012). Construcción y uso de tipologías: movilidad geográfica en la frontera México-Estados Unidos. En M. Ariza y L. Velasco (Coords.), Métodos cualitativos y su aplicación empirica. Por los caminos de la investigación sobre migración internacional (pp. 419-454). México, Distrito Federal: Instituto de Investigaciones Sociales-unam, El Colegio de la Frontera Norte. 
Jaquet, H. (2008). Más allá de la frontera, las fronteras: una aproximación socioespacial a las situaciones fronterizas de Misiones, Argentina. En H. Dilla (Coord.), Ciudades en la frontera. Aproximaciones críticas a los complejos urbanos transfronterizos (pp. 33-66). Santo Domingo, República Dominicana: Editorial Manatí.

Jessop, B. (2004). La economía política de la escala y la construcción de las regiones fronterizas. EURE, 30(89), 25-41.

Kolossov, V. (2005). Border Studies: Changing Perspectives and Theoretical Approaches. Geopolitics, 10(4), 606-632.

Konrad, V. (2015). Toward a Theory of Borders in Motion. Journal of Borderlands Studies, 30(1), 1-17. doi: 10.1080/08865655.2015.1008387

Kralich, S., Benedetti, A. y Salizzi, E. (2012). Aglomeraciones transfronterizas y movilidad. Una aproximación desde casos sudamericanos. Boletim Gaúcho de Geografía, (38), 111-136.

Machado, L. O. (2005). Estado, territorialidade, redes. Cidades-gêmeas na zona de fronteira sul-americana. En M. Silveira (Org.), Continente em chamas. Globalização e território na América Latina (pp. 243-284). Río de Janeiro, Brasil: Civilização Brasileira.

Mallimaci, A. I. (2012). Movilidades y permanencias. Repensando la figura del movimiento en las migraciones. Revista de Temas de Antropología y Migración, (3), 77-92.

Marques, R. y Machado, T. (2008). O mérito das cidades-gêmeas nos espaços fronteiriços. Observatorio Iberoamericano del Desarrollo Local y la Economía Social, 2(5), 22-30.

Morales, A. (2010). Desentrañando fronteras y sus movimientos transnacionales entre pequeños estados. Una aproximación desde la frontera Nicaragua-Costa Rica. En M. E. Anguiano y A. M. López (Eds.), Migraciones y frontera. Nuevos contornos para la movilidad internacional (pp. 185-224). Barcelona, España: Icaria.

Newby, C. A. (2006). Border Crossing and Settlement in El Paso, Texas: Understanding Transborder Actors. Annual Meeting, Montreal.

Newman, D. (2015). Revisiting good fences and neighbours in a postmodern world after twenty years: theoretical reflections on the state of contemporary border studies. Nordia Geographical Publications, 44(4), 13-19.

Nuñez, A. (2014). Bipolaridad fronteriza: dialéctica entre globalización, privatización del Estado y la territorialidad de la nación. Chile, siglo xxi. En M. Tapia y A. González (Eds.), Regiones fronterizas. Migración y los desafios para los Estados nacionales latinoamericanos (pp. 73-95). Santiago, Chile: RIL Editores.

Ovando, C. y Ramos, R. (2016). Imaginarios geográficos en torno a la franja fronteriza de Tarapacá: el Estado y los habitantes/migrantes. Scripta Nova. Revista electrónica de Geografía y Ciencias Sociales, xx(529), 1-25.

Parella, S. (2014). Una exploración de las prácticas transfronterizas en la zona urbana Caléxico (Estados Unidos)-Mexicali. En M. Tapia y A. González (Eds.), Regiones fronterizas, migración y los desafíos para los estados nacionales latinoamericanos (pp. 41-69). Santiago, Chile: RIL Editores.

Parker, N. y Vaughan-Williams, N. (2009). Lines in the Sand? Towards an Agenda for Critical Border Studies. Geopolitics, 14(3), 582-587.

Perkmann, M. (2003). Cross-Border Regions in Europe: Significance and Drivers of Regional Cross-Border Co-Operation. European Urban and Regional Studies, 10(2), 153-171. doi:10.1177/0969776403010002004 
Portes, A., Guarnizo, L. y Landolt, P. (2003). La globalización desde abajo: transnacionalismo inmigrante y desarrollo. La experiencia de Estados Unidos y América Latina. México: FLACSO-México, Miguel Ángel Porrúa.

Ratto, S. (2001). El debate sobre la frontera a partir de Turner. La new western history, los borderlans y el estudio de las fronteras en América Latina. Boletín del Instituto de Historia Argentina y Americana "Dr. Emilio Ravignan", (24), 105-125.

Ribas, N. (2011). Río Bravo Mediterráneo. Las regiones fronterizas en la época de la globalización. Barcelona, España: Edicions Bellaterra.

Sassen, S. (2007). Una sociología de la globalización. Buenos Aires, Argentina: Kast.

Sassen, S. (2010). Territorio, autoridad y derechos. De los ensamblajes medievales a los ensamblajes globales. Madrid, España: Katz Editores.

Shen, J. (2014). Not quite a twin city: Cross-boundary integration in Hong Kong and Shenzhen. Habitat International, 42, 138-146.

Soriano, R. y Fuentes, M. (2015). La vulnerabilidad de las mujeres porteadoras de Ceuta y Melilla como sujeto de derechos humanos. En A. Karla y S. Izcara (Eds.), Migrantes, transmigrantes, deportados y derechos humanos: un enfoque binacional (pp. 117-140). México Distrito Federal: Editorial de Fontamara.

Steiman, R. (2012). Zona de fronteira e cidades gemeas: Uma tipologia das interacoes transfronteiricas. En C. E. Zárate (Ed.), Espacios urbanos y sociedades transfronterizas en la Amazonia (pp. 154-166). Leticia, Colombia: Universidad Nacional de Colombia.

Tapia, M. (2014). Extranjeros fronterizos en las regiones extremas de Chile: entre migración y circulación 1990-2014. En N. Rojas y J. T. Vicuña (Eds.), Migración y trabajo. Estudio y propuestas para la inclusión sociolaboral de migrantes en Arica (pp. 31-55). Santiago, Chile: Ciudadano Global-oim.

Tapia, M. y González, A. (2014). Presentación. Fronteras, regiones fronterizas y migraciones. Entre apertura, integración y cierre. En M. Tapia y A. González (Eds.), Regiones fronterizas, migración y los desafíos para los Estados nacionales latinoamericanos (pp. 15-38). Santiago, Chile: RIL Editores.

Tapia, M. y Parella, S. (2015). Las regiones fronterizas para el estudio de la migración y la circulación. Un análisis de dos casos ilustrativos. En M. Guizardi (Ed.), Las fronteras del transnacionalismo. Limites y desbordes de la experiencia migrante en el centro y norte de Chile (pp. 173-206). Chile: Ocho Libros.

Velde, M. van der y Naerssen, T. van (2011). People, Borders, Trajectories: an Approach to Cross-Border Mobility and Immobility in and to the European Union. Area, 43(2), 1-7. doi:10.1111/j.1475-4762.2010.00974.x

Wang, D. (2004). Hong Kongers' Cross-Border Consumption and Shopping in Shenzhen: Patterns and Motivations. Journal of Retailing and Consumer Services, 11(3), 149-159.

Zapata-Barrero, R. y Ferrer-Gallardo, X. (2012). Las fronteras en la época de la movilidad. En R. Zapata-Barrero y X. Ferrer-Gallardo (Eds.), Fronteras en movimiento. Migraciones hacia la Unión Europea en el contexto Mediterráneo (pp. 11-56). Barcelona, España: Edicions Bellaterra.

Zimmerbauer, K. (2011). Conceptualizing Borders in Cross-Border Regions: Case Studies of the Barents and Ireland-Wales Supranational Regions. Journal of Borderlands Studies, 26(2), 211-229. 
Zuñiga, V. (2009). Tratando de entender la sociedad fronteriza: recorrido crítico de los estudios mexicanos sobre la cultura en la frontera México-Estados Unidos (1976-2000). En C. Salazar (Ed.), Migraciones contemporáneas. Contribución al debate (pp. 69-84). Bolivia: Corporación Andina de Fomento, CIDEs-umsa, Plural editores.

Zusman, P. (1999). Representaciones, imaginarios y conceptos en torno a la producción material de las fronteras a partir del debate Hevilla-Escamilla. Revista Bibliográfica de Geografía y Ciencias Sociales, 4(149).

Marcela Aurora Tapia Ladino

Chilena. Licenciada en Educación con mención en Historia y Geografía y Profesora de Historia y Geografía por la Universidad de Concepción. Magíster en Historia en la Universidad de Concepción. Doctor en América Latina Contemporánea por el Instituto de Investigación Universitaria Ortega y Gasset y la Universidad Complutense de Madrid. Líneas de investigación: migración, movilidad y frontera. Entre sus publicaciones más recientes: Tapia, M., Liberona, N. y Contreras, Y. (2017). El surgimiento de un territorio circulatorio en la frontera chileno-peruana: estudio de las prácticas socio-espaciales fronterizas. Geografia Norte Grande, (66), 117-141. 